\title{
Double spacing multi-wavelength L-band Brillouin erbium fiber laser with Raman pump
}

\begin{abstract}
A new multi-wavelength Brillouin erbium fiber laser (BEFL), which operates in the L-band region with double frequency Brillouin spacing, is demonstrated. This design uses a Raman pump (RP) and a piece of $2 \mathbb{\mathbb { k l m }}$ highly nonlinear fiber as a gain medium. The double frequency spacing is achieved by employing a dual ring configuration, which is formed by utilizing a four-port circulator that removes the odd-order Stoke signals. Twenty Stokes and seventeen anti-Stokes lines, which have optical signal to noise ratio (OSNR) greater than $15 \mathrm{ddB}$, are generated simultaneously with a spacing of 0.16 $\mathrm{flm}$ when Brillouin pump and RP powers were fixed at the optimum values of $8 \mathrm{dBm}$ and 40 Th $\mathrm{W}$, respectively. The BEFL can be tuned in the range between $1591 \mathrm{frlm}$ to $1618 \mathrm{Fllm}$. The proposed configuration increases the number of lines generated and the OSNR, and thus allows a compact multi-wavelength laser source to be realized.
\end{abstract}

Keyword: Brillouin erbium fiber laser; Double frequency spacing; Highly nonlinear fiber; Multi-wavelength laser 